\title{
Cellulose Nanocrystal (CNC) Capsules from Oil Palm Empty Fruit Bunches (OPEFB)
}

\author{
Felycia Edi Soetaredjo ${ }^{1, *}{ }^{\mathbb{D}}$, Shella Permatasari Santoso ${ }^{1 \mathbb{D}}$, Gladdy L. Waworuntu ${ }^{2}$, Farida Laniwati \\ Darsono $^{3}$ \\ 1 Department of Chemical Engineering, Widya Mandala Surabaya Catholic University, Kalijudan 37, Surabaya 60114 , \\ Indonesia \\ 2 Faculty of Medicine. Widya Mandala Surabaya Catholic University, Kalisari Selatan No. 1, Surabaya 60112, Indonesia \\ 3 Faculty of Pharmacy, Widya Mandala Surabaya Catholic University, Kalisari Selatan No. 1, Surabaya 60112, Indonesia \\ * Corresponding author: felyciae@yahoo.com;
}

Scopus Author ID 25960403800

Received: 8.02.2021; Revised: 25.04.2021; Accepted: 1.05.2021; Published: 10.06.2021

\begin{abstract}
The raw material used for this NCC production was oil palm empty bunches (OPEFB), currently waste from oil palm plantations. Delignification of OPEFB was carried out using $2 \mathrm{~N} \mathrm{NaOH}$ solution at $70^{\circ} \mathrm{C}$ for 6 hours. NCC maximum yield from OPEFB is $62.1 \%$ (dry cellulose basis) obtained at a concentration of $54 \%$ sulfuric acid and a temperature of $50^{\circ} \mathrm{C}$. The addition of plasticizers (glycerol and PEG) reduced the elastic modulus of NCC capsules from 7951.4 MPa to $4758 \mathrm{MPa}$ (glycerol) and 3225 (PEG). The addition of glycerol and PEG did not affect the disintegration time of NCC capsules. NCC capsules have a release capability similar to the commercially available gelatin capsules. At 14 minutes, discharge reached about 43\%, and it becomes constant after 18 minutes.
\end{abstract}

Keywords: nanocrystalline cellulose; capsules; gelatin.

(C) 2021 by the authors. This article is an open-access article distributed under the terms and conditions of the Creative Commons Attribution (CC BY) license (https://creativecommons.org/licenses/by/4.0/).

\section{Introduction}

Capsules are widely used to transfer powder drugs into the human body, and they should be colorless and odorless. The primary raw material for making capsules is gelatin [1]. Gelatin is a chemical obtained from collagen, which comes from various animal byproducts. Gelatin available in the market is divided into two types: those made from young pork skin (gelatin type A) or those made from bones and old skin (gelatin type B). The preparation of type A gelatin is relatively much easier and cheaper than type B gelatin.

The use of gelatin as raw material for capsules or other pharmaceutical products creates controversy considering that the raw material source is often unclear, whether it comes from pigs or other animals (especially for countries where most of the population is Muslim). Besides the source of gelatin raw material, which is often unclear, another obstacle often faced with gelatin as a raw material for making capsules is the solubility of this compound in water. Some of the gelatin in the capsule will be difficult to dissolve in water due to the cross-linking reaction. This cross-linking reaction can occur due to many factors, such as aldehyde compounds in capsule fillers, storage at high temperatures and humidity, etc. [1]. The different gelatin preparation will also cause the structure and composition to vary so that the capsule's dissolution becomes uncontrolled.

Various kinds of natural polymers have been investigated further into potential raw materials to manufacture capsules. Alternative raw materials that have been studied include 
sago starch [2], starch [3], sodium alginate, and starch [4]. The problem faced in using these raw materials for the manufacture of capsules is their low mechanical properties. This study develops alternative materials with more advantages than existing raw materials to overcome the various weaknesses in commercial capsule-making raw materials (gelatin) and other alternative materials.

Nanocrystalline cellulose (NCC) is a form of cellulose with impressive mechanical properties, good biocompatibility properties, and is renewable. NCC is a potential biomaterial for pharmaceuticals and medicine [5-19]. In a previous study by Wijaya et al. [20], the solution's pH controlled the release of drugs from NCC. However, NCC direct use as a drug carrier has several drawbacks; the drug release cannot be adequately controlled because NCC disintegration is rapid. To overcome these weaknesses, it is necessary to modify the NCC in capsule form so that the drug absorption and release can be controlled and takes place efficiently. In this study, NCC from oil palm empty fruit bunches (OPEFB) is used as raw material for making capsules to replace gelatin.

\section{Materials and Methods}

\subsection{Materials.}

OPEFB used in this study was obtained from a palm plantation located in Kotawaringin Timur, Central Kalimantan, Indonesia. Glycerol (CAS Number 56-81-5, purity > 99.5\%) and polyethylene glycol (PEG) (CAS Number: 25322-68-3) were purchased from Sigma-Aldrich.

\subsection{Delignification of OPEFB.}

Before the delignification process was carried out, OPEFB was cut into small pieces and dried in the oven for 24 hours until the moisture content was less than $15 \%$. The dried OPEFB was then reduced to a size of 60/80 mesh. The delignification OPEFB was carried out using chemical and radiation processes using microwaves. The lignin removal process was carried out using $2 \mathrm{~N} \mathrm{NaOH}$ solution at $70^{\circ} \mathrm{C}$ for 6 hours. After that, the radiation process using microwaves was carried out for 10 minutes with a power of 700 watts. After the lignin removal process was completed, the remaining cellulose was separated from the solution and washed using reverse osmosis water. Then the cellulose was dried until the water content is less than $10 \%$.

\subsection{Hydrolysis of OPEFB cellulose into NCC.}

Hydrolysis of cellulose into cellulose nanocrystals was carried out using sulfuric acid at various $40-60 \%$ concentrations at temperature variations of 40 to $50^{\circ} \mathrm{C}$. The time used for the hydrolysis process was 60 minutes. During the hydrolysis process, stirring was carried out at a speed of $600 \mathrm{rpm}$. The cellulose suspension was added $4^{\circ} \mathrm{C}$ cold water to stop the hydrolysis reaction; the mixture was left to stand for 12 hours to separate between the clear and cloudy layers. The cloudy layer was then transferred to the dialysis tube, and the dialysis process was carried out for four days. The nanocrystalline cellulose formed was then separated by centrifugation and dried using a freeze dryer. 


\subsection{Preparation of NCC capsules.}

NCC, glycerol, and polyethylene glycol (PEG) were mixed in a specific ratio. The mixture was then dissolved in reverse osmosis water and stirred at a stirring speed of 500 RPM for 45 minutes at $50 \mathrm{oC}$. Capsules were made by dipping a stainless steel mold into the mixture for 6-10 seconds. The layer attached to the mold was dried in a humidity chamber at $37^{\circ} \mathrm{C}$ and $50 \%$ humidity. The dried capsules were then removed from the mold and cut into standard capsule sizes.

\subsection{Characterization.}

SEM analysis was used to observe nanocrystalline cellulose morphology (JEOL JSM6390 field emission SEM operated at an acceleration voltage of $5 \mathrm{kV}$ ). Before SEM analysis, nanocrystalline cellulose samples were coated with a thin layer of conductive platinum using a fine auto coater (JFC-1200, JEOL, Ltd., Japan) for 120 seconds in an argon atmosphere. XRD analysis was performed to investigate nanocrystalline cellulose crystallinity (Philips PANalytical X'Pert X-ray powder diffractometer). The diffractogram was obtained at $40 \mathrm{kV}$, $30 \mathrm{~mA}$, and a range of $0.05^{\circ} / \mathrm{s}$.

NCC capsules characterization was carried out by measuring the modulus of elasticity, swelling property, and capsule disintegration time. The modulus of elasticity of the capsules was measured using a nanoindenter at five different points. The swelling property measurements were carried out in a phosphate buffer ( $\mathrm{pH} 3$ ) with the following working steps: the capsules were immersed in a phosphate buffer solution for a specific time (10 to 60 minutes), then the capsule surface was immediately dried using a filter paper to remove water on the capsule surface. After that, the capsules were weighed immediately. The following equation calculates the swelling percentage:

$$
S P=\frac{W_{s}-W_{D}}{W_{D}} \times 100 \%
$$

Ws is the mass of the capsule after immersion, and WD is the mass of the capsule when it is dry.

\subsection{Release of amoxicillin from NCC capsules.}

The release experiment of amoxicillin from nanocrystalline cellulose was carried out in vitro at $37^{\circ} \mathrm{C}$ in a phosphate buffer solution. The amount of drug released into the buffer solution was measured as a function of time.

\section{Results and Discussion}

\subsection{Preparation of NCC and NCC capsules.}

In making capsules from OPEFB, the delignification process plays an essential role in making NCC successful. The chemical composition of OPEFB before and after the delignification process can be seen in Table 1. Determination of the chemical composition of OPEFB before and after the delignification process was carried out using the thermal gravimetric analysis (TGA) method. Table 1 shows that the delignification process of OPEFB is effective; most of the lignin and hemicellulose content can be degraded well. 
Table 1. Chemical composition of OPEFB before and after the delignification process.

\begin{tabular}{l|c|c|c} 
Composition & Before delignification, \% & After delignification, \% & TGA temperature, ${ }^{\mathbf{}} \mathbf{C}$ \\
\hline water & 8.9 & 7.8 & $50-200$ \\
\hline hemicellulose & 27.8 & 7.4 & $200-300$ \\
\hline Lignin & 26.7 & 3.6 & $300-360$ \\
\hline cellulose & 31.4 & 77.8 & $360-500$ \\
\hline Ash & 5.2 & 3.4 & $>500$
\end{tabular}

The manufacture of NCC from lignocellulosic materials requires two main process steps. The initial step is removing lignin, which is usually carried out through various pretreatment processes, both chemically and physically [21-25]. In this initial step, most of the lignin and some hemicellulose can be dissolved and removed from the cellulose skeleton. The next step is acid hydrolysis of the cellulose fibers to form a colloidal suspension of cellulose crystals. In this acid hydrolysis process, the amorphous part of cellulose fibers will be hydrolyzed to glucose, while the crystal fragments remain intact due to their excellent stability under acidic conditions.

NCC maximum yield from OPEFB is $62.1 \%$ (dry cellulose basis) obtained at a concentration of $54 \%$ sulfuric acid and a temperature of $50^{\circ} \mathrm{C}$. The ratio of cellulose and sulfuric acid is 1:10 with a hydrolysis time of 60 minutes. These high yields indicate that the cellulose fraction of oil palm empty bunches contain more crystalline regions than amorphous ones. During the hydrolysis process using sulfuric acid, the amorphous regions of cellulose fibers were broken down to glucose, and the crystalline portion remains intact due to their excellent stability in the sulfuric acid environment.

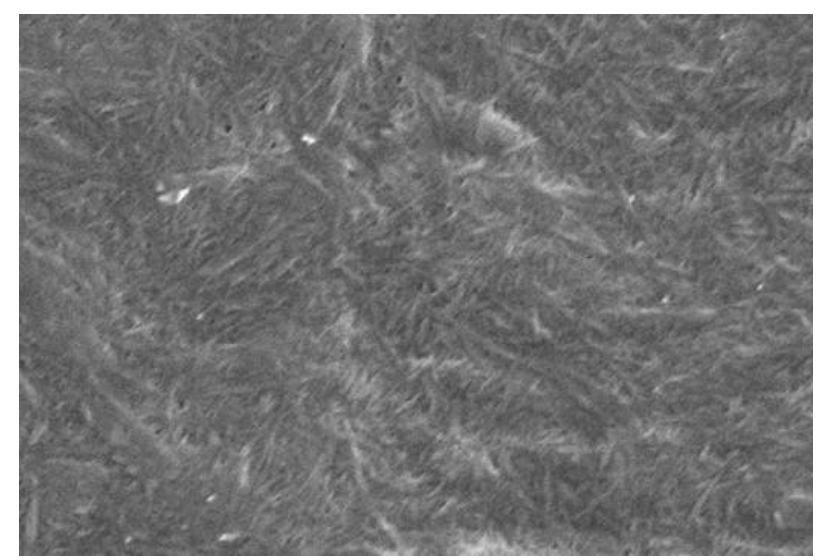

Figure 1. SEM image of NCC from OPEFB (x 10,000, 5.0 kV, WD $11.7 \mathrm{~mm}$ ).

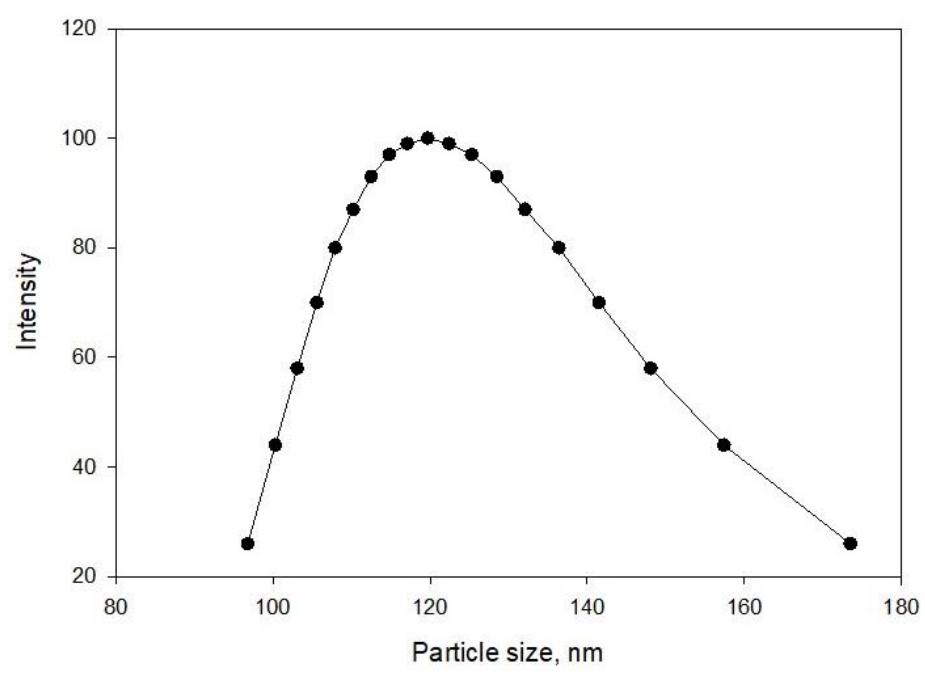

Figure 2. The particle size distribution of NCC from OPEFB. 


\subsection{Characterization of NCC and NCC capsules.}

Figure 1 shows the surface topography of the NCC produced from oil palm empty bunches. From SEM observations, it can be seen that NCC has a morphology like a stem. As previously mentioned, this rod-like morphology is typical for NCC. The NCC particle size distribution is shown in Figure 2. The NCC particle size of the empty oil palm bunches was around $108-171.5 \mathrm{~nm}$, with a median size of $142.7 \mathrm{~nm}$ and an average of $149.4 \mathrm{~nm}$.

NCC X-ray diffraction pattern (XRD) from OPEFB and standard crystal cellulose I from JCPDS No. 00-050-22411 are depicted in Figure 3. The XRD CNC pattern shows four distinct peaks at $2 \theta=15.3^{\circ}, 16.5^{\circ}, 22.4^{\circ}$, and $35.2^{\circ}$, which correspond to the planes 110,110 , 200, and 004. These peaks are located at the same position as the standard crystal peaks of cellulose I, 15.9॰ $(110), 16.4 \circ(110), 22.6^{\circ}(200)$, and 34.6॰ (004).

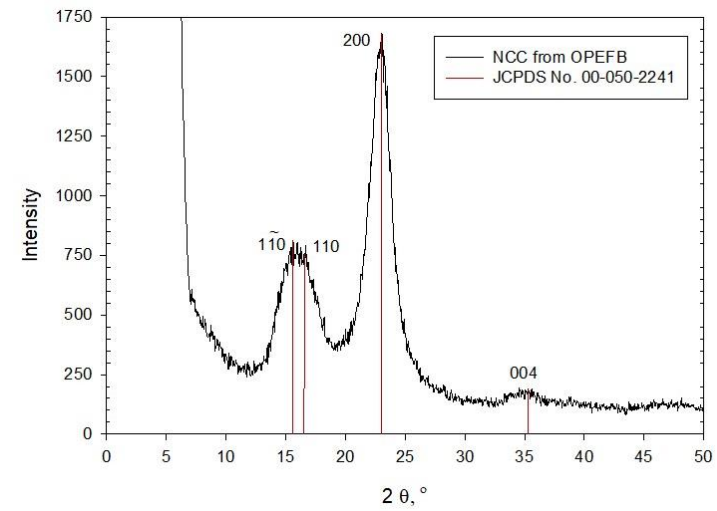

Figure 3. XRD diffractogram of NCC from OPEFB.
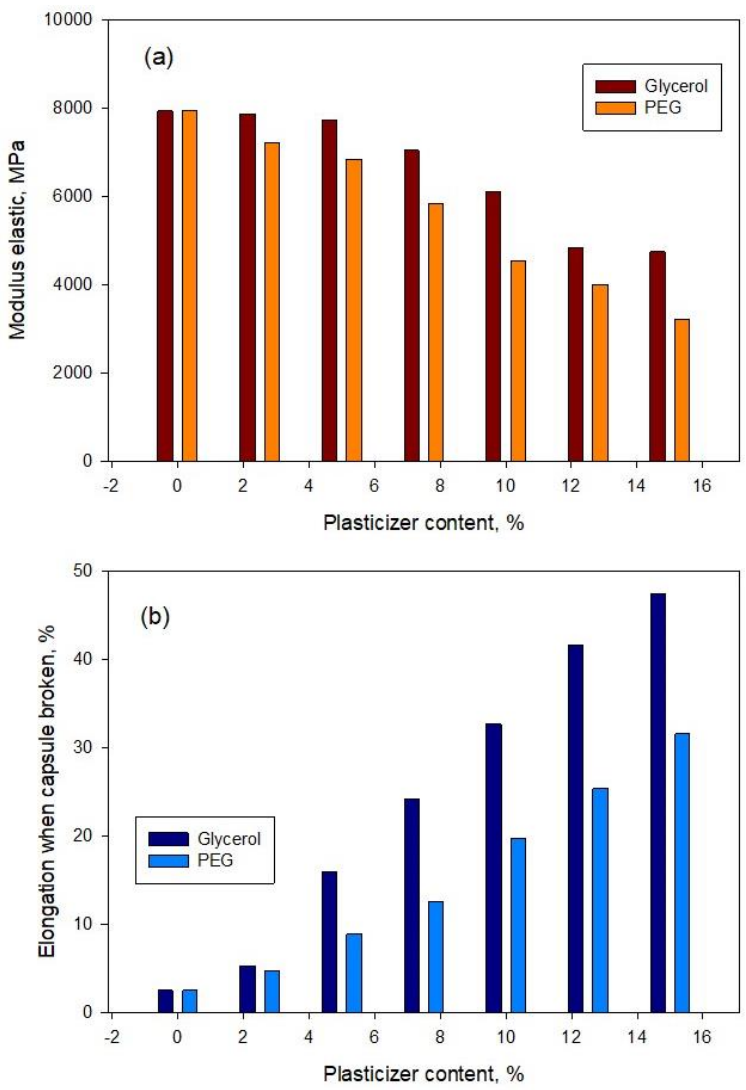

Figure 4. The influence of plasticizer on modulus elastic and elongation of NCC capsule.

Glycerol and PEG function as plasticizers in NCC capsules. The effect of adding glycerol and PEG on the NCC capsule's mechanical properties was expressed in terms of elastic modulus and elongation when the NCC capsule was broken. The elastic modulus of NCC of 
OPEFB is 7951.4 MPa. The effect of adding glycerol and PEG (2.5 - 15\%) on the elastic modulus and elongation of the NCC capsule can be seen in Figure 4.

In Figure 4 (a), it can be seen that the addition of plasticizers (glycerol and PEG) reduced the elastic modulus of NCC capsules from $7951.4 \mathrm{MPa}$ to $4758 \mathrm{MPa}$ (glycerol) and 3225 (PEG). These plasticizers also affect the elongation when the NCC capsule is broken; the percent elongation increases with the increase in the percentage of plasticizer added (Figure 4 (b)). Figure 4 shows that glycerol provides a better plasticizing effect than PEG; this is because glycerol has a smaller molecular size than PEG, so that it is more easily absorbed on the NCC surface. The same results were obtained by Zhang et al. [1].

Swelling Properties (SP) measurements of NCC capsules were carried out in phosphate buffer ( $\mathrm{pH} 3$ ). The addition of glycerol and PEG will increase the SP of the NCC capsule (Figure 5). PEG molecules have many strong hydrophilic ether oxygen bonds; these bonds lead to more significant hydrophilic properties. The increase in PEG percentage increases the NCC capsule's hydrophilic properties so that the SP will increase, as shown in Figure 5. NCC capsules with PEG 15\% have a higher SP than NCC capsules with 5\% PEG.

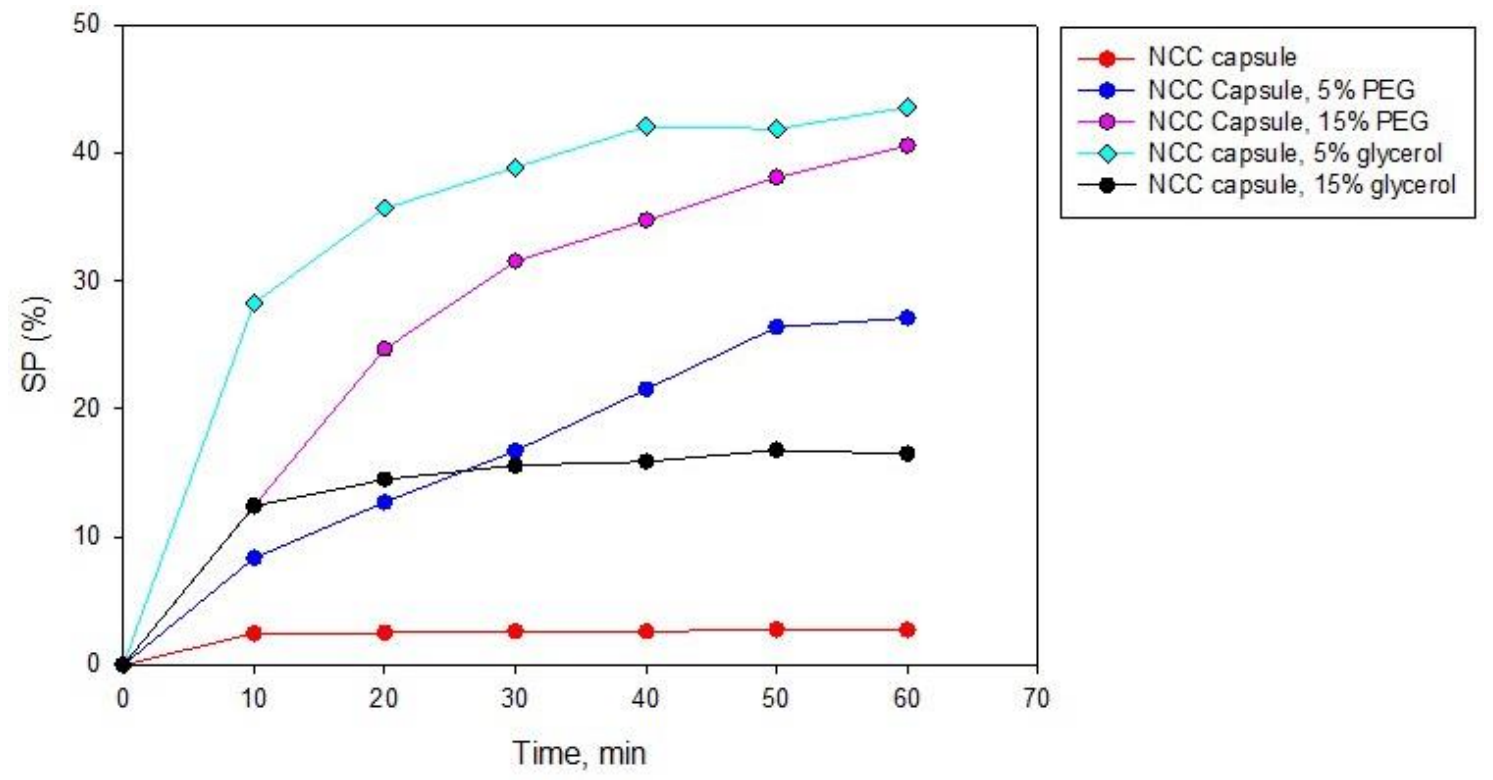

Figure 5. The influence of plasticizer on swelling properties of NCC capsules.

Glycerol has a different PEG behavior; increasing glycerol concentration from 5\% to $15 \%$ causes a significant decrease in SP. The decrease in SP with increasing glycerol concentrations is due to the increased binding strength between glycerol and NCC particles, which inhibits water molecules' entry into the NCC capsule [1]. The smallest SP was obtained at the addition of $15 \%$ glycerol. As previously explained, the glycerol molecule is smaller than PEG, so it is easily absorbed on the NCC surface, which causes the binding strength to increase, which will inhibit the entry of water molecules into the NCC capsule [1].

\subsection{Disintegration time and drug release.}

The disintegration time is an essential parameter for the application of NCC capsules on a commercial scale. After entering the stomach, the NCC capsule must be destroyed before the human body can absorb the drug. From the experimental results obtained, the NCC capsules or NCC capsules with the addition of glycerol or PEG will begin to disintegrate in about 3 minutes and are destroyed in the 5th minute. The addition of glycerol and PEG did not affect the disintegration time of NCC capsules. 
For drug-releasing trials, amoxicillin was used. The amoxicillin concentration released into the phosphate buffer solution was measured using a UV spectrophotometer at a maximum wavelength of $228 \mathrm{~nm}$. Commercially available gelatin capsules were used as a comparison. The percent release of amoxicillin in a phosphate buffer solution can be seen in Figure 6. Figure 6 shows that the NCC capsules have a release capability similar to the commercially available gelatin capsules. At 14 minutes, discharge reached about 43\%, and it sloped down after 18 minutes.

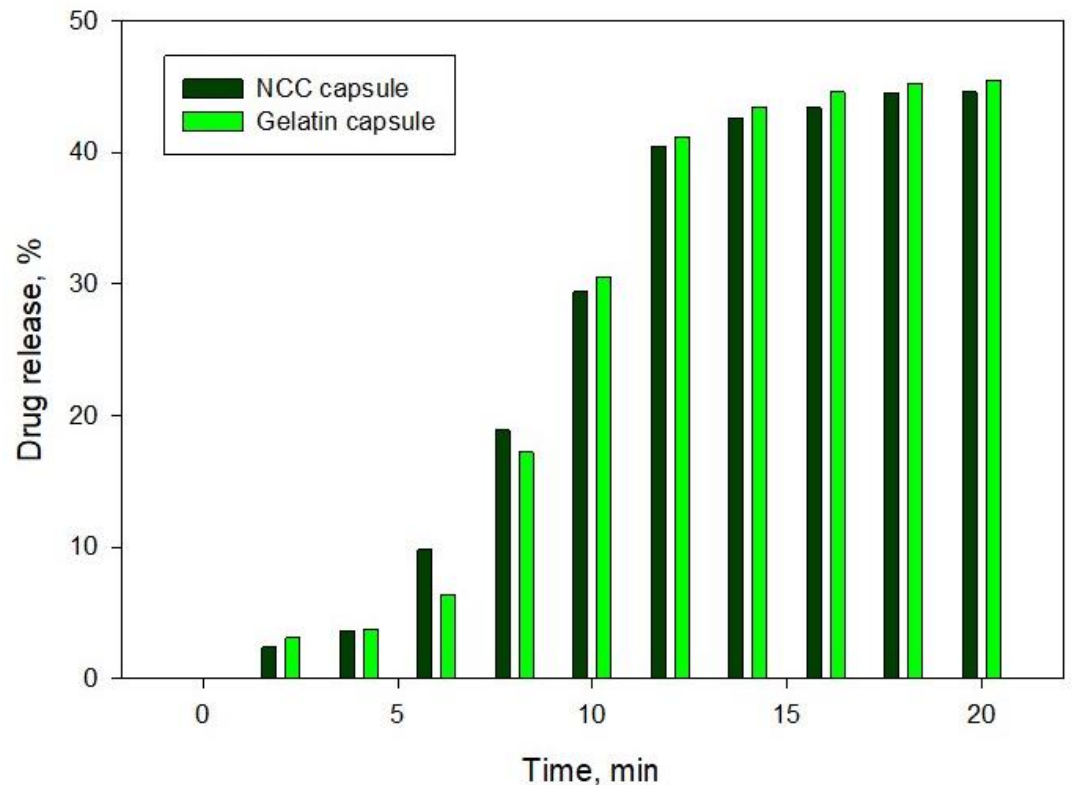

Figure 6. The percent release of amoxicillin in a phosphate buffer solution for NCC and gelatin capsules.

\section{Conclusions}

Oil palm empty bunches have been successfully used as raw material for the manufacture of NCC capsules. The manufacturing process for NCC capsules is straightforward and can be applied easily on an industrial scale. The effect of glycerol and polyethylene glycol (PEG) plasticizers on the mechanical properties, break time, and swelling performance (SP) of NCC capsules have also been studied. The elastic modulus and elongation of the NCC capsule rupture time were affected by the addition of glycerol and PEG. The best swelling performance is given by the NCC capsule with the addition of $5 \%$ glycerol. The addition of glycerol and PEG did not affect the breakdown time of NCC capsules. NCC capsules have a drug release capability that is almost equivalent to gelatin capsules on the market.

\section{Funding}

This research was funded by Widya Mandala Surabaya Catholic University Research Grant no: 233a/WM01.5/N/2020.

\section{Acknowledgments}

This research has no acknowledgment.

\section{Conflicts of Interest}

The authors declare no conflict of interest. 


\section{References}

1. Zhang, Y.; Zhao, Q.; Wang, H.; Jiang, X.; Cha, R. Preparation of green and gelatin-free nanocrystalline cellulose capsules. Carbohydr. Polym. 2017, 164, 358-363, https://doi.org/10.1016/j.carbpol.2017.01.096.

2. Misale, B.V.; Gavali, H.B.M.; Katare, S.D.; Yadav, A.V. Sago starch capsule shell: A suitable alternative to gelatin capsule shells. Indian J. Pharm. Educ. Res. 2008, 42, 48-52.

3. Mali, S.; Sakanaka, L.S.; Yamashita, F.; Grossmann, M.V.E. Water sorption and mechanical properties of cassava starch films and their relation to plasticizing effect. Carbohydr. Polym. 2005, 60, 283-289, https://doi.org/10.1016/j.carbpol.2005.01.003.

4. Lu, C.L.; Feng, Q.; Chen, H.; Liu, L.P. Preparation and performance test of vegetable enteric hollow capsules by one-line molding process. Chinese Pharm. J. 2015, 50, 2057-2063.

5. Bayoumi, A.; Sarg, M.T.; Fahmy, T.Y.A.; Mohamed, N.F.; El-Zawawy, W.K. The behavior of natural biomass materials as drug carriers in releasing loaded Gentamicin sulphate. Arab. J. Chem. 2020, 13, 89208934, https://doi.org/10.1016/j.arabjc.2020.10.018.

6. Murizan, N.I.S.; Mustafa, N.S.; Ngadiman, N.H.A.; Yusof, N.M.; Idris, A. Review on nanocrystalline cellulose in bone tissue engineering applications. Polymers 2020, 12, https://doi.org/10.3390/polym12122818.

7. Gupta, R.D.; Raghav, N. Differential effect of surfactants tetra-n-butyl ammonium bromide and N-Cetyl-N, $\mathrm{N}, \mathrm{N}$-trimethyl ammonium bromide bound to nano-cellulose on binding and sustained release of some nonsteroidal anti-inflammatory drugs. Int. J. Biol. Macromol. 2020, 164, 2745-2752, https://doi.org/10.1016/j.ijbiomac.2020.08.091.

8. Jadhav, S.; Kaur, A.; Bansal, A.K. Comparison of downstream processing of nanocrystalline solid dispersion and nanosuspension of diclofenac acid to develop solid oral dosage form. Pharmaceutics 2020, 12, 1-24, https://doi.org/10.3390/pharmaceutics12111015.

9. Foo, M.L.; Ooi, C.W.; Tan, K.W.; Chew, I.M.L. A Step Closer to Sustainable Industrial Production: Tailor the Properties of Nanocrystalline Cellulose from Oil Palm Empty Fruit Bunch. J. Env. Chem. Eng. 2020, 8, https://doi.org/10.1016/j.jece.2020.104058.

10. Farooq Adil, S.; Bhat, V.S.; Batoo, K.M.; Imran, A.; Assal, M.E.; Madhusudhan, B.; Khan, M., Al-Warthan, A. Isolation and characterization of nanocrystalline cellulose from flaxseed Hull: A future onco-drug delivery agent. J. Saudi Chem. Soc. 2020, 24, 374-379, https://doi.org/10.1016/j.jscs.2020.03.002.

11. Gupta, R.D.; Raghav, N. Nano-crystalline cellulose: Preparation, modification and usage as sustained release drug delivery excipient for some non-steroidal anti-inflammatory drugs. Int. J. Biol. Macromol. 2020, 147, 921-930, https://doi.org/10.1016/j.ijbiomac.2019.10.057.

12. Bundjaja, V.; Sari, T.M.; Soetaredjo, F.E.; Yuliana, M.; Angkawijaya, A.E.; Ismadji, S.; Cheng, K.-C., Santoso, S.P. Aqueous sorption of tetracycline using rarasaponin-modified nanocrystalline cellulose. J. Mol. Liq. 2020, 301, https://doi.org/10.1016/j.molliq.2019.112433.

13. Salimi, S.; Sotudeh-Gharebagh, R.; Zarghami, R.; Chan, S.Y.; Yuen, K.H. Production of Nanocellulose and Its Applications in Drug Delivery: A Critical Review. ACS Sustain. Chem. Eng. 2019, 7, 15800-15827, https://doi.org/10.1021/acssuschemeng.9b02744.

14. Foo, M.L.; Tan, C.R.; Lim, P.D.; Ooi, C.W.; Tan, K.W.; Chew, I.M.L. Surface-modified nanocrystalline cellulose from oil palm empty fruit bunch for effective binding of curcumin. Int. J. Biol. Macromol. 2019, 138, 1064-1071, https://doi.org/10.1016/j.ijbiomac.2019.07.035.

15. Karimian, A.; Parsian, H.; Majidinia, M.; Rahimi, M.; Mir, S.M.; Samadi Kafil, H.; Shafiei-Irannejad, V.; Kheyrollah, M.; Ostadi, H., Yousefi, B. Nanocrystalline cellulose: Preparation, physicochemical properties, and applications in drug delivery systems. Int. J. Biol. Macromol. 2019, 133, 850-859, https://doi.org/10.1016/j.ijbiomac.2019.04.117.

16. Akhavan-Kharazian, N.; Izadi-Vasafi, H. Preparation and characterization of chitosan/gelatin/nanocrystalline cellulose/calcium peroxide films for potential wound dressing applications. Int. J. Biol. Macromol. 2019, 133, 881-891, https://doi.org/10.1016/j.ijbiomac.2019.04.159.

17. Putro, J.N.; Ismadji, S.; Gunarto, C.; Yuliana, M.; Santoso, S.P.; Soetaredjo, F.E., Ju, Y.H. The effect of surfactants modification on nanocrystalline cellulose for paclitaxel loading and release study. J. Mol. Liq. 2019, 282, 407-414, https://doi.org/10.1016/j.molliq.2019.03.037.

18. Karimi-Dehkordi, N.; Minaiyan, M.; Talebi, A.; Akbari, V.; Taheri, A. Nanocrystalline cellulose-hyaluronic acid composite enriched with GM-CSF loaded chitosan nanoparticles for enhanced wound healing. Biomed. Mater. 2019, 14, https://doi.org/10.1088/1748-605x/ab026c.

19. Xie, J.; Luo, Y.; Liu, Y.; Ma, Y.; Yue, P.; Yang, M. Novel redispersible nanosuspensions stabilized by coprocessed nanocrystalline cellulose-Sodium carboxymethyl starch for enhancing dissolution and oral bioavailability of baicalin. Int. J. Nanomedicine 2019, 14, 353-369, https://doi.org/10.2147/IJN.S184374.

20. Wijaya, C.J.; Saputra, S.N.; Soetaredjo, F.E.; Putro, J.N.; Lin, C.X.; Kurniawan, A.; Ju, Y.H.; Ismadji, S. Cellulose nanocrystals from passion fruit peels waste as antibiotic drug carrier. Carbohydr. Polym. 2017, 175, 370-376, https://doi.org/10.1016/j.carbpol.2017.08.004. 
21. Oh, Y.; Park, S.; Jung, D.; Oh, K.K.; Lee, S.H. Effect of hydrogen bond donor on the choline chloride-based deep eutectic solvent-mediated extraction of lignin from pine wood. Int. J. Biol. Macromol. 2020, 165, 187197, https://doi.org/10.1016/j.ijbiomac.2020.09.145.

22. Zakaria, S.M.; Idris, A.; Chandrasekaram, K.; Alias, Y. Efficiency of bronsted acidic ionic liquids in the dissolution and depolymerization of lignin from rice husk into high value-added products. Ind. Crops Prod. 2020, 157, https://doi.org/10.1016/j.indcrop.2020.112885.

23. Davaritouchaee, M.; Chen, S.; Mancini, R.J. Delignification and Enzyme-Diffusion Kinetics of Radical Systems Treating Wheat Straw. Ind. Eng. Chem. Res. 2020, 59, 20656-20666, https://doi.org/10.1021/acs.iecr.0c04107.

24. Wang, J.-X.; Asano, S.; Kudo, S.; Hayashi, J.-I. Deep Delignification of Woody Biomass by Repeated Mild Alkaline Treatments with Pressurized $\mathrm{O}_{2}$. ACS Omega 2020, 5, 29168-29176, https://doi.org/10.1021/acsomega.0c03953.

25. Okur, M.; Eslek-Koyuncu, D.D. Investigation of pretreatment parameters in the delignification of paddy husks with deep eutectic solvents. Biomass Bioenergy 2020, 142, https://doi.org/10.1016/j.biombioe.2020.105811. 